\title{
Induction chemotherapy in head and neck squamous cell carcinoma of the paranasal sinus and nasal cavity: a role in organ preservation
}

Chan-Young Ock ${ }^{1}$, Bhumsuk Keam ${ }^{1,2}$, Tae Min Kim ${ }^{1,2}$, Doo Hee Han ${ }^{3}$, Tae-Bin Won ${ }^{3}$, Se-Hoon Lee ${ }^{1,2}$, J. Hun Hah³, Tack-Kyun Kwon ${ }^{3}$, Dong-Wan Kim ${ }^{1,2}$, Dong-Young Kim³, Chae-Seo Rhee ${ }^{3}$, Hong-Gyun $\mathrm{Wu}^{4}$, Myung-Whun Sung ${ }^{3}$, and Dae Seog Heo ${ }^{1,2}$

\begin{abstract}
${ }^{1}$ Department of Internal Medicine, Seoul National University Hospital, Seoul; ${ }^{2}$ Cancer Research Institute, Seoul National University College of Medicine, Seoul; Departments of ${ }^{3}$ Otorhinolaryngology and ${ }^{4}$ Radiation Oncology, Seoul National University Hospital, Seoul, Korea
\end{abstract}

\author{
Received: January 28, 2015 \\ Revised : April 30, 2015 \\ Accepted: May 12, 2015

\section{Correspondence to} \\ Bhumsuk Keam, M.D. \\ Department of Internal Medicine, \\ Seoul National University Hos- \\ pital, 101 Daehak-ro, Jongno-gu, \\ Seoul 03080, Korea \\ Tel: +82-2-2072-7215 \\ Fax: +82-2-2072-7379 \\ E-mail: bhumsuk@snu.ac.kr
}

Background/Aims: The role of induction chemotherapy (IC) for eyeball preservation has not been established in head and neck squamous cell carcinoma (HNSCC) of the paranasal sinus and nasal cavity (PNSNC). Periorbital involvement frequently leads to eyeball exenteration with a margin of safety. We evaluated the treatment outcomes, including survival and eyeball preservation, of patients who received IC for HNSCC of the PNSNC.

Methods: We reviewed 21 patients diagnosed with HNSCC of the PNSNC who were treated with IC. We analyzed response, eyeball preservation rate, and overall survival.

Results: Tumors were located in the paranasal sinus $(n=14)$ or nasal cavity $(n=7)$. Most patients had stage $T_{4} a(n=10)$ or $T_{4} b(n=7)$ disease. More than half of the patients received a chemotherapy regimen of docetaxel, fluorouracil, and cisplatin $(\mathrm{n}=11)$. Thirteen patients $(61.9 \%)$ achieved a partial response after IC and 15 patients (71.4\%) achieved T down-staging. Among 17 patients with stage $\mathrm{T}_{4}$ disease, which confers a high risk of orbital exenteration, 14 (82.4\%) achieved preservation of the involved eye. The 3-year overall survival (OS) rate of patients who achieved a partial response to IC was $84.6 \%$. The 3-year OS rate of patients with stable disease or disease progression after IC was $25.0 \%(p=0.038)$.

Conclusions: IC could be considered for down-staging patients with advanced T-stage disease. It could also be a reasonable option for eyeball preservation in locally advanced HNSCC of the PNSNC.

Keywords: Induction chemotherapy; Head and neck neoplasms; Paranasal sinuses; Nasal cavity; Orbit evisceration

\section{INTRODUCTION}

Head and neck squamous cell carcinoma (HNSCC) accounts for nearly $10 \%$ of all malignancies, and paranasal sinus and nasal cavity (PNSNC) neoplasms represent 3\% of head and neck malignancies [1,2]. Although tumors of the PNSNC are relatively rare, treatment planning for cancer of the PNSNC is challenging because of its heterogenous behavior and proximity to vital structures such as the orbit, skull base, and brain. Historically, the preferred treatment for tumors of the PNSNC, as well as HNSCC of other sites, consisted of en bloc resection of 
the primary tumor. This method has been replaced by less-invasive but equally effective treatments such as definitive radiotherapy and concurrent chemoradiotherapy (CCRT) [3-6]. Despite the availability of such advanced modalities, the overall survival (OS) rate of HNSCC of the PNSNC has not improved and the 5-year survival rate has remained approximately $40 \%$ to $50 \%$ [7-10]. Moreover, the risk of orbital invasion and the need for eyeball exenteration represent extreme deterioration of quality of life for patients with HNSCC of the PNSNC. Approximately $12 \%$ to $40 \%$ of patients with locally advanced HNSCC of the PNSNC eventually undergo eyeball exenteration [11-14].

The benefits of induction chemotherapy were first noted from evidence that suggested that chemotherapy improved the organ preservation rate and reduced distant metastasis in laryngeal cancer [15,16]. Although most studies of induction chemotherapy in HNSCC have focused on larynx preservation, some attempts have been made to establish the effects of induction chemotherapy on eyeball preservation in cancer of the PNSNC, which confers a high risk of orbital invasion. To date, encouraging organ preservation rates have been reported [1719], but actual orbital preservation rates could be better than reported since the most effective chemotherapy regimen was not used in these previous studies; the regimen of docetaxel, cisplatin, and fluorouracil, which is more effective than previous regimens, is now the standard of care and is used frequently [20-22]. We previously reported treatment outcomes of definitive radiotherapy in patients diagnosed with HNSCC of the PNSNC at Seoul National University Hospital (SNUH) and we showed that patients treated with induction chemotherapy had favorable OS compared to those who were not treated with chemotherapy $(p=0.037)$ [23]. In a recent retrospective study of induction chemotherapy containing a taxane and platinum in HNSCC of the PNSNC, $67 \%$ of patients achieved at least a partial response and only six out of 46 patients failed to preserve the involved eye [24]. These findings imply that adequate induction chemotherapy can save the involved eye in patients with orbital invasion.

The purpose of the current study was to evaluate the role of induction chemotherapy in locally advanced HNSCC of the PNSNC in terms of survival and organ preservation rates.

\section{METHODS}

\section{Study population}

We retrospectively reviewed medical records of patients who were diagnosed with HNSCC of the PNSNC who were treated at SNUH from August 2005 to March 2012. For the analysis, we included adult patients aged 18 years or older with primary squamous carcinoma of the PNSNC who were treated with induction chemotherapy. Other inclusion criteria included having more than one measurable lesion according to the response evaluation criteria in solid tumours (RECIST) 1.1 [25]; an Easte ern Cooperative Oncology Group performance status (ECOG PS) of o to 2; and adequate hematologic, hepatic, and renal functions. We analyzed the following variables during a review of the medical records of included patients: sex; diagnosis date; age; ECOG PS; history of other malignancies; tumor location; TNM stage according to the American Joint Committee on Cancer Staging Manual, 7 th edition, which defined stage $\mathrm{T}_{3}$ as a tumor invading the orbital floor, stage $\mathrm{T} 4 \mathrm{a}$ as a tumor invading the orbital contents, and stage $\mathrm{T} 4 \mathrm{~b}$ as a tumor invading the orbital apex [26]; induction regimen; induction response; whether a salvage operation was performed; whether the eyeball was preserved; and the date of last follow-up or death.

\section{Treatment}

The treatment modality was decided by a multidisciplinary team. Bulky nodal status, advanced T- or $\mathrm{N}$-stage, and the possibility of eyeball preservation after induction chemotherapy influenced the treatment decision. Induction chemotherapy was cycled every 3 weeks and induction regimens included docetaxel, cisplatin, and fluorouracil. Following induction chemotherapy, patients received definitive treatment such as CCRT, radiotherapy alone, and operation. CCRT regimens consisted of conventional standard fractionated radiotherapy of more than 60 Gy for primary tumor and regional lymph nodes, with concurrent chemotherapy with weekly administration of cisplatin $35 \mathrm{mg} / \mathrm{m}^{2}$.

\section{Response evaluation and outcome measurement}

We assessed complete physical examinations and all imaging studies, including magnetic resonance images or computed tomography of the head and neck, for all 
patients. Follow-up imaging was performed after two $(\mathrm{n}=8)$ or three $(\mathrm{n}=13)$ cycles of induction chemotherapy and responses were evaluated according to RECIST version $1.1[25]$. T down-staging was defined as a reducfi tion of $\mathrm{T}$-stage from $\mathrm{T}_{4} \mathrm{~b}$ to $\mathrm{T}_{4} \mathrm{a}$ or $\mathrm{T}_{4} \mathrm{a}$ to a lower stage. The primary objective of our study was to quantify the eyeball preservation rate and OS according to response to induction chemotherapy. Eyeball preservation was defined as eyeball function that was not disrupted by salvage operation, including eyeball exenteration, or progression of an inoperable tumor. A tumor was determined to be inoperable by a multidisciplinary team that included surgeons and radiologists [11-14]. Follow-up imaging was performed at 4 to 8 weeks after the end of definitive treatment, then every 3 to 6 months until progression or death. OS was measured from the date of diagnosis until death or the date of last follow-up, if censored.

\section{Safety and compliance assessment}

Adverse events were monitored and recorded every cycle of induction chemotherapy according to Common Terminology Criteria for Adverse Events version 3.o. Compliance of definitive treatment of CCRT or RT was defined as good if patient received more than $90 \%$ of pre-planned dose of radiotherapy.

\section{Statistical analysis}

We used the chi-square test to determine the associations between response to chemotherapy and clinicopathologic parameters. We also conducted Kaplan-Meier estimates and Cox regression analyses of OS. Results were considered significant when $p$ values were less than o.05. Analyses were completed with STATA software version 12 (StataCorp LP, College Station, TX, USA).

\section{Ethics}

The study protocol was approved by the Institutional Review Board of SNUH (approval number: H-1307-051504) and was conducted in accordance with the Principles of the Declaration of Helsinki.

\section{RESULTS}

\section{Patient characteristics}

Baseline characteristics of patients are listed in Table 1. The median follow-up duration was 83.1 months (range, 28.8 to 109.1). The median age was 57 years (range, 24 to 75$)$ and 15 of the patients (71.4\%) were male. Tumors were located in the paranasal sinus $(n=14)$ or the nasal cavity $(n=7)$. Most patients had stage T4a $(n=10)$ or $\mathrm{T}_{4} \mathrm{~b}(\mathrm{n}=7)$ disease. Induction chemotherapy regimens were docetaxel plus flourouracil plus cisplatin (n $=11)$, docetaxel plus flourouracil $(\mathrm{n}=8)$, and docetaxel plus cisplatin $(\mathrm{n}=2)$. Fifteen patients $(71.4 \%)$ received three cycles of induction chemotherapy and six patients (28.6\%) received two cycles. Mean cycle of induction chemotherapy was 2.7. Thirteen patients $(61.9 \%)$ achieved a partial response after induction chemotherapy and 15 patients (71.4\%) achieved $\mathrm{T}$ down-staging. Toxicity profile during induction chemotherapy was summarized in Table 2. Overall, induction chemotherapy was tolerable with except nausea and neutropenia (grade 3 or 4: $14.3 \%$ and $9.5 \%$, respectively). Sixteen patients (76.2\%) tolerated full-dose of induction chemotherapy, but four patients (19\%) and one patient (4.8\%) had $80 \%$ and $75 \%$ of pre-planned dose, respectively. However, all patients had good compliance during the definitive treatment of CCRT or RT.

After induction chemotherapy, 14 patients underwent definitive radiotherapy either with concurrent chemotherapy with weekly administration of cisplatin $(\mathrm{n}=11)$ or radiotherapy alone $(n=3)$. Six patients underwent a salvage operation and one patient was lost to follow-up after induction chemotherapy.

Patients who achieved either stable disease or progressive disease after induction chemotherapy were older, had higher T-stages, and received more salvage operations than patients who achieved a partial response, but these differences were not statistically significant. All patients who achieved a partial response also achieved $\mathrm{T}$ down-staging and eyeball preservation. Six out of eight patients with stable or progressive disease did not achieve $\mathrm{T}$ down-staging, and three patients eventually lost an eyeball during the follow-up period.

\section{Eyeball preservation after induction chemotherapy}

Patients with HNSCC of the PNSNC who had stage T4a 
Ock CY, et al. Induction chemotherapy in PNSNC cancer

Table 1. Patient characteristics

\begin{tabular}{|c|c|c|c|c|}
\hline Characteristic & $\operatorname{All}(\mathrm{n}=21)$ & Induction $\mathrm{PR}(\mathrm{n}=13)$ & Induction $\mathrm{SD} / \mathrm{PD}(\mathrm{n}=8)$ & $p$ value \\
\hline Age, yr & $57(24-75)$ & $56(24-73)$ & $61(50-75)$ & 0.282 \\
\hline Male sex & $15(71.4)$ & $8(61.5)$ & $7(87.5)$ & 0.201 \\
\hline \multicolumn{5}{|l|}{ ECOG PS } \\
\hline o & $7(33 \cdot 3)$ & $3(23.1)$ & $4(50.0)$ & \\
\hline 1 & $12(57.1)$ & $8(61.5)$ & $4(50.0)$ & \\
\hline 2 & $2(9.5)$ & $2(15 \cdot 4)$ & o & 0.298 \\
\hline \multicolumn{5}{|l|}{ T-stage } \\
\hline 1 & $1(4.7)$ & $1(7.7)$ & o & \\
\hline 2 & o & o & o & \\
\hline 3 & $3(14.3)$ & $3(23.1)$ & o & \\
\hline $4 \mathrm{a}$ & $10(47.6)$ & $4(30.8)$ & $6(75.0)$ & \\
\hline $4 b$ & $7(33 \cdot 3)$ & $5(38.5)$ & $2(25.0)$ & 0.190 \\
\hline \multicolumn{5}{|l|}{$\mathrm{N}$-stage } \\
\hline o & $14(66.7)$ & $9(69.2)$ & $5(62.5)$ & \\
\hline 1 & $3(14 \cdot 3)$ & $2(15.4)$ & $1(12.5)$ & \\
\hline 2 & $4(19.1)$ & $2(15.4)$ & $2(25.0)$ & 0.859 \\
\hline \multicolumn{5}{|l|}{ Stage } \\
\hline III & $3(14 \cdot 3)$ & $3(23.1)$ & o & \\
\hline IV & $18(85.7)$ & $10(76.9)$ & $8(100.0)$ & 0.142 \\
\hline \multicolumn{5}{|l|}{ Tumor location } \\
\hline Paranasal sinus & $14(66.7)$ & $9(69.2)$ & $5(62.5)$ & \\
\hline Nasal cavity & $7(33.3)$ & $4(30.8)$ & $3(37.5)$ & 0.751 \\
\hline \multicolumn{5}{|c|}{ Induction chemotherapy regimen } \\
\hline DFP & $11(52.4)$ & $7(53.9)$ & $4(50.0)$ & \\
\hline FP & $8(38.1)$ & $5(38.5)$ & $3(37.5)$ & \\
\hline DP & $2(9.5)$ & $1(7.7)$ & $1(12.5)$ & 0.935 \\
\hline \multicolumn{5}{|c|}{ Induction chemotherapy response } \\
\hline CR & o & o & o & \\
\hline PR & $13(61.9)$ & $13(100.0)$ & o & \\
\hline $\mathrm{SD}$ & $6(28.6)$ & o & $6(75.0)$ & \\
\hline $\mathrm{PD}$ & $2(9.5)$ & o & $2(25.0)$ & $<0.001^{a}$ \\
\hline \multicolumn{5}{|l|}{ T down-staging } \\
\hline Yes & $15(71.4)$ & $13(100.0)$ & $2(25.0)$ & \\
\hline No & $6(28.6)$ & o & $6(75.0)$ & $<0.001^{\mathrm{a}}$ \\
\hline \multicolumn{5}{|c|}{ Definitive treatment } \\
\hline CCRT & $11(52.4)$ & $7(53.9)$ & $4(50.0)$ & \\
\hline Radiotherapy & $3(14 \cdot 3)$ & $1(7.7)$ & $2(25.0)$ & \\
\hline Operation $^{\mathrm{b}}$ & $6(28.5)$ & $4(30.8)$ & $2(25.0)$ & \\
\hline No treatment & $1(4.8)$ & $1(7.7)$ & o & 0.631 \\
\hline \multicolumn{5}{|l|}{ Salvage operation ${ }^{c}$} \\
\hline Yes & $10(47.6)$ & $5(38.5)$ & $5(62.5)$ & \\
\hline No & $11(52.4)$ & $8(61.5)$ & $3(37.5)$ & 0.284 \\
\hline
\end{tabular}


Table 1. Continued

\begin{tabular}{|c|c|c|c|c|}
\hline Characteristic & $\operatorname{All}(\mathrm{n}=21)$ & Induction $\mathrm{PR}(\mathrm{n}=13)$ & Induction $\mathrm{SD} / \mathrm{PD}(\mathrm{n}=8)$ & $p$ value \\
\hline \multicolumn{5}{|c|}{ Eyeball preservation } \\
\hline Yes & $18(85.7)$ & $13(100.0)$ & $5(62.5)$ & \\
\hline No & $3(14 \cdot 3)$ & o & $3(37.5)$ & $0.017^{\mathrm{a}}$ \\
\hline
\end{tabular}

Values are presented as median (range) or number (\%).

PR, partial response; SD, stable disease; PD, progressive disease; ECOG PS, Eastern Cooperative Oncology Group performance status; DFP, docetaxel plus fluorouracil plus cisplatin; FP, fluorouracil plus cisplatin; DP, docetaxel plus cisplatin; CR, complete response; CCRT, concurrent chemoradiotherapy.

${ }^{a}$ Values indicate statistically significant correlations with $p$ values less than 0.05 .

${ }^{\mathrm{b}}$ Patients assigned to surgical removal of tumor immediately after induction chemotherapy.

${ }^{\mathrm{c}}$ Patients assigned to surgery immediately after induction chemotherapy or who needed salvage operation after definitive treatment with CCRT or radiotherapy.

Table 2. Toxicity profile of induction chemotherapy

\begin{tabular}{|c|c|c|c|c|}
\hline & Grade 1 & Grade 2 & Grade 3 & Grade 4 \\
\hline Nausea and vomiting & $2(9 \cdot 5)$ & $5(23.8)$ & $3(14 \cdot 3)$ & 0 \\
\hline Diarrhea & 0 & $3(14 \cdot 3)$ & 0 & 0 \\
\hline Neuropathy & $3(14 \cdot 3)$ & o & $\mathrm{o}$ & $\mathrm{O}$ \\
\hline Neutropenia & 0 & $2(9 \cdot 5)$ & $2(9 \cdot 5)$ & 0 \\
\hline Anemia & $\mathrm{O}$ & $4(19.0)$ & o & 0 \\
\hline Thromcytopenia & $1(4.8)$ & 0 & ० & 0 \\
\hline Others & $3(14 \cdot 3)$ & $1(4.8)$ & $\mathrm{O}$ & 0 \\
\hline
\end{tabular}

Values are presented as number (\%). Grade of toxicity was referred from Common Terminology Criteria for Adverse Events (CTCAE) version 3.0.

or $\mathrm{T}_{4} \mathrm{~b}$ disease were at risk of orbital penetration [13,14]. All of the patients who had a T-stage lower than $\mathrm{T}_{4}(\mathrm{n}=$ 4) preserved their eyeballs after induction chemotherapy (Fig. 1). However, half of the patients (5 out of 10) with stage T4a disease required a salvage operation and two of these patients lost an eyeball: one underwent orbital exenteration and the other had a tumor that was inoperable due to massive invasion of the orbital cavity. More than half of the patients with stage $\mathrm{T}_{4} \mathrm{~b}$ disease (4 out of 7) also underwent a salvage operation; only one of these patients underwent orbital exenteration.

\section{Survival according to response to induction chemo- therapy}

During the follow-up period, 3- and 5-year OS rates of patients with a partial response to induction chemotherapy were $84.6 \%$ and $65.8 \%$, respectively. In patients with stable or progressive disease, the 3- and 5-year OS rates were both $25 \%(p=0.036)$ (Fig. $2 A)$. Patients who achieved $\mathrm{T}$ down-staging had longer OS than those who did not achieve T down-staging (3-year OS, 80\% vs. 16.7\%; 5-year OS, $64 \%$ vs. $16.7 \%$; $p=0.027$ ) (Fig. 2 B). OS according to stage and N-stage were graphed in Supplementary Fig. 1. Patients with N-stage more than 1 had poorer OS than No disease (3-year OS, $71.4 \%$ vs. $42.9 \%$; $p=0.031$ ).

\section{DISCUSSION}

In the current study, we observed beneficial effects of induction chemotherapy in squamous cell carcinoma of the PNSNC on organ preservation and OS according to response to chemotherapy. Of the 21 patients included in our analysis, three patients who did not respond to induction chemotherapy eventually lost the involved eyeball. However, all patients who achieved a partial response or $\mathrm{T}$ down-staging after induction chemotherapy preserved the eyeball and achieved a favorable overall prognosis.

Although the role of induction chemotherapy has 
been well-established in organ preservation $[15,16]$, the survival benefits from induction chemotherapy have been controversial in locally advanced HNSCC. Randomized phase III trials that compared induction chemotherapy before definitive CCRT and CCRT alone, which is the standard treatment regimen for locally advanced HNSCC, failed to show any survival benefits of adding induction chemotherapy [27-29]. Although a

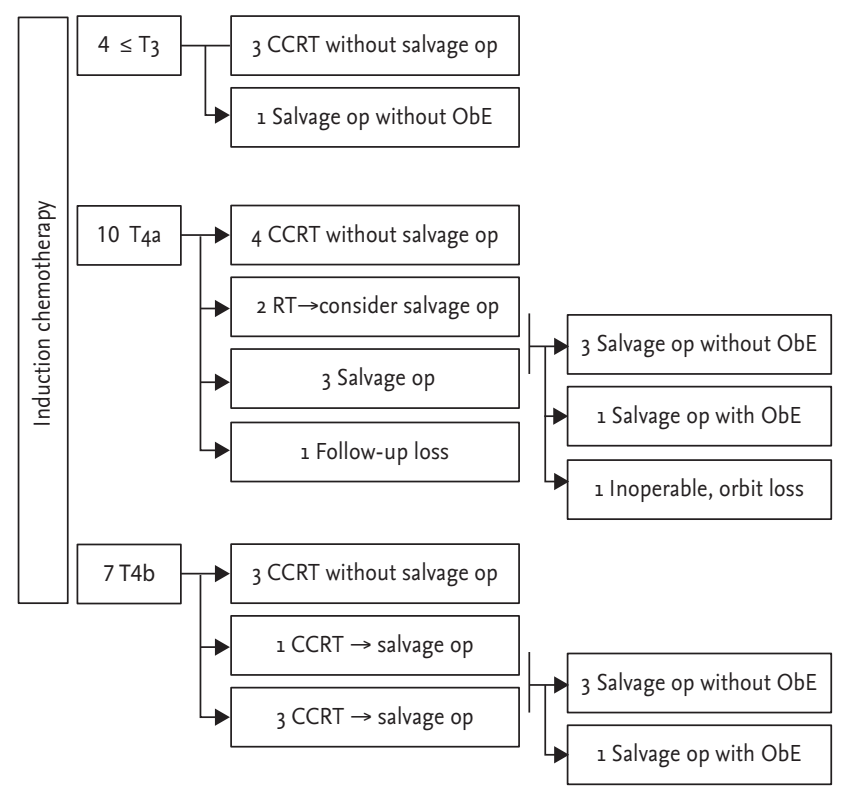

Figure 1. Flow diagram of patients included in the study. Of 17 patients with stage $\mathrm{T}_{4}$ disease, only three patients lost an eyeball during the follow-up period. CCRT, concurrent chemo radiotherapy; op, operation; ObE, orbital exenteration; $\mathrm{RT}$, radiotherapy.

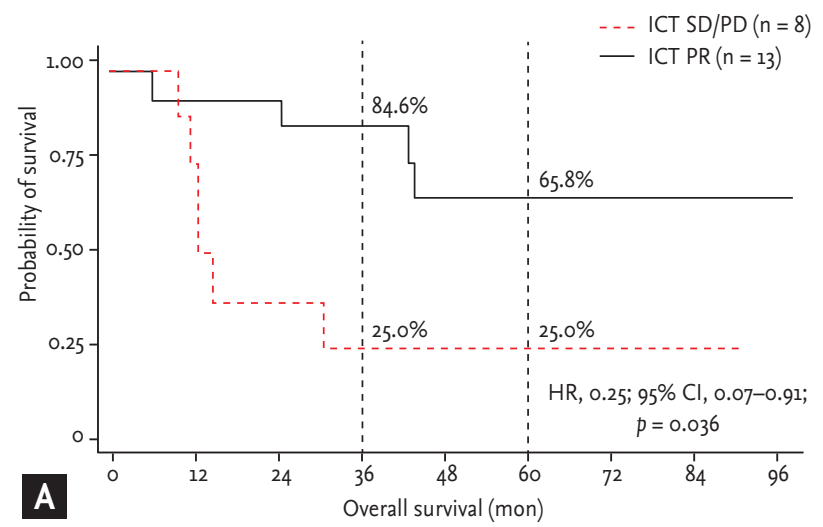

recent trial showed significant OS benefits of induction chemotherapy, the benefits were false positives since the survival benefits were abolished in patients who were assigned to cisplatin as the CCRT regimen and in patients with oropharyngeal cancer, which composed more than half of the cohort [30]. Considering the low incidence of tumors arising from the PNSNC, patients with PNSNC tumors were not included in previous studies and there have been no published prospective trials regarding the effects of induction chemotherapy on survival in $\mathrm{HN}$ SCC of the PNSNC.

In the current study, we observed survival outcomes after induction chemotherapy in patients with tumors arising from the PNSNC. A total of $61.9 \%$ of patients achieved a partial response to induction chemotherapy and the 3-year OS rate of these patients was $84.6 \%$. This result is comparable to results of a previous study, which showed a response rate of $67 \%$ and a 2-year OS rate of $77 \%$ in patients who achieved either partial response or stable disease [24]. An important finding of the current study is that all patients who achieved a partial response to induction chemotherapy were saved from orbital exenteration, which might have been required in patients with stage $T_{4}$ disease $(n=9)$ if induction chemotherapy had not been administered. However, eight out of 21 patients $(38.1 \%)$ failed to achieve a partial response to chemotherapy and the 3-year OS rate of these patients was significantly lower $(25 \%)$ than patients who achieved a partial response. Additionally, these patients suffered a higher risk of orbital loss either by orbital

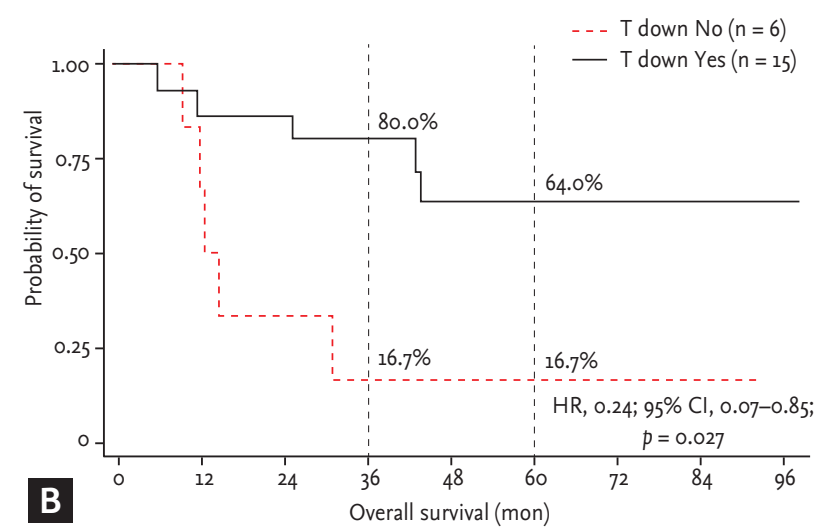

Figure 2. Kaplan-Meier curves of patients according to response to induction chemotherapy (A) and T down-staging after induction chemotherapy (B). Numbers in the graph indicate the probability of survival at 3 years and at 5 years. HR, hazard ratio; CI, confidence interval; ICT, induction chemotherapy; SD, stable disease; PD, progressive disease; PR, partial response; T down, T down-staging. 
exenteration $(\mathrm{n}=2)$ or inoperable eyeball invasion $(\mathrm{n}=$ 1). Previous reports have presented advances in surgical techniques and adjuvant radiotherapy that may improve eyeball preservation [31,32], but induction chemotherapy could be considered for increasing eyeball preservation rates in patients with advanced T-stage HNSCC of the PNSNC.

Previous trials have shown that patients who complete induction chemotherapy, as noted by an analysis of intention-to-treat cohorts, benefit from chemotherapy [29]. Moreover, in a comparison of survival outcomes of adding induction chemotherapy to definitive CCRT in our institution, a subgroup analysis revealed that patients who achieved at least a partial response attained significant survival benefits compared to patients who received CCRT alone (unpublished). Therefore, it is important to select patients who would tolerate and benefit from induction chemotherapy. However, it is difficult to determine who would benefit from induction chemotherapy since we observed no significant differences in clinicopathologic characteristics between patients who achieved a partial response and patients with stable disease or progressive disease in the current study.

This study has several limitations. The small number of patients included in the analysis does not allow for significant differences to be discerned between subgroups according to response to induction chemotherapy or orbital preservation. Moreover, the current study had a retrospective design, so the included patients might not be representative cases of all diagnosed patients. For example, the cohort may have included a high number of healthy patients who tolerated induction chemotherapy, which might have resulted in better outcomes than expected. Nevertheless, considering the very low incidence of HNSCC of the PNSNC and the results of the current study, we conclude that induction chemotherapy may offer improved organ preservation and favorable survival. We focused on the effects of induction chemotherapy for this analysis, and the timing of salvage surgery and surgical techniques were not established. These factors might have affected the final outcomes of organ preservation.

In conclusion, patients with HNSCC of the PNSNC, especially advanced T-stage disease, who responded to induction chemotherapy experienced increased rates of organ preservation and OS. Well-designed prospective trials are needed to establish the efficacy of induction chemotherapy in tumors arising from the PNSNC.

\section{KEY MESSAGE}

1. Induction chemotherapy in the paranasal sinus and nasal cavity cancer showed overall response rate of $61.9 \%$.

2. In high risk of orbital exenteration, $82.4 \%$ achieved preservation of the eye due to induction chemotherapy.

3. Induction chemotherapy is effective for down-staging patients with advanced T-stage.

\section{Conflict of interest}

No potential conflict of interest relevant to this article was reported.

\section{Acknowledgments}

This study was supported by the Seoul National University Hospital Research Fund (Grant No. 30-2013-0070).

\section{REFERENCES}

1. Siegel R, Naishadham D, Jemal A. Cancer statistics, 2012. CA Cancer J Clin 2012;62:10-29.

2. Batsakis JG. Tumours of the Head and Neck: Clinical and Pathological Considerations. 2nd ed. Baltimore: Lippincott Williams and Wilkins, 1979.

3. Pignon JP, le Maitre A, Maillard E, Bourhis J; MACHNC Collaborative Group. Meta-analysis of chemotherapy in head and neck cancer (MACH-NC): an update on 93 randomised trials and 17,346 patients. Radiother Oncol 2009;92:4-14.

4. Haddad RI, Shin DM. Recent advances in head and neck cancer. N Engl J Med 2008;359:1143-1154.

5. Forastiere AA, Goepfert H, Maor M, et al. Concurrent chemotherapy and radiotherapy for organ preservation in advanced laryngeal cancer. N Engl J Med 2003;349:20912098.

6. Tobias JS, Monson K, Gupta N, et al. Chemoradiotherapy for locally advanced head and neck cancer: 10-year follow-up of the UK Head and Neck (UKHANı) trial. Lancet Oncol 2010;11:66-74. 
7. Dulguerov P, Jacobsen MS, Allal AS, Lehmann W, Calcaterra T. Nasal and paranasal sinus carcinoma: are we making progress? A series of 220 patients and a systematic review. Cancer 2001;92:3012-3029.

8. Ganly I, Patel SG, Singh B, et al. Craniofacial resection for malignant paranasal sinus tumors: report of an international collaborative study. Head Neck 2005;27:575-584.

9. Guntinas-Lichius O, Kreppel MP, Stuetzer H, Semrau R, Eckel HE, Mueller RP. Single modality and multimodality treatment of nasal and paranasal sinuses cancer: a single institution experience of 229 patients. Eur J Surg Oncol 2007;33:222-228.

10. Snyers A, Janssens GO, Twickler MB, et al. Malignant tumors of the nasal cavity and paranasal sinuses: longterm outcome and morbidity with emphasis on hypothalamic-pituitary deficiency. Int J Radiat Oncol Biol Phys 2009;73:1343-1351.

11. Ahmad K, Cordoba RB, Fayos JV. Squamous cell carcinoma of the maxillary sinus. Arch Otolaryngol 1981;107:4851.

12. Marchetta FC, Sako K, Mattick WL, Stinziano GD. Squamous cell carcinoma of the maxillary antrum. Am J Surg 1969;118:805-807.

13. McCary WS, Levine PA, Cantrell RW. Preservation of the eye in the treatment of sinonasal malignant neoplasms with orbital involvement: a confirmation of the original treatise. Arch Otolaryngol Head Neck Surg 1996;122:657659.

14. Perry C, Levine PA, Williamson BR, Cantrell RW. Preservation of the eye in paranasal sinus cancer surgery. Arch Otolaryngol Head Neck Surg 1988;114:632-634.

15. The Department of Veterans Affairs Laryngeal Cancer Study Group. Induction chemotherapy plus radiation compared with surgery plus radiation in patients with advanced laryngeal cancer. N Engl J Med 1991;324:16851690.

16. Lefebvre JL, Chevalier D, Luboinski B, Kirkpatrick A, Collette L, Sahmoud T. Larynx preservation in pyriform sinus cancer: preliminary results of a European Organization for Research and Treatment of Cancer phase III trial: EORTC Head and Neck Cancer Cooperative Group. J Natl Cancer Inst 1996;88:890-899.

17. Lee YY, Dimery IW, Van Tassel P, De Pena C, Blacklock JB, Goepfert H. Superselective intra-arterial chemotherapy of advanced paranasal sinus tumors. Arch Otolaryngol Head Neck Surg 1989;115:503-511.
18. Rosen A, Vokes EE, Scher N, Haraf D, Weichselbaum RR, Panje WR. Locoregionally advanced paranasal sinus carcinoma: favorable survival with multimodality therapy. Arch Otolaryngol Head Neck Surg 1993;119:743-746.

19. Papadimitrakopoulou VA, Ginsberg LE, Garden AS, et al. Intraarterial cisplatin with intravenous paclitaxel and ifosfamide as an organ-preservation approach in patients with paranasal sinus carcinoma. Cancer 2003;98:22142223.

20. Hitt R, Lopez-Pousa A, Martinez-Trufero J, et al. Phase III study comparing cisplatin plus fluorouracil to paclitaxel, cisplatin, and fluorouracil induction chemotherapy followed by chemoradiotherapy in locally advanced head and neck cancer. J Clin Oncol 2005;23:8636-8645.

21. Vermorken JB, Remenar E, van Herpen C, et al. Cisplatin, fluorouracil, and docetaxel in unresectable head and neck cancer. N Engl J Med 2007;357:1695-1704.

22. Posner MR, Hershock DM, Blajman CR, et al. Cisplatin and fluorouracil alone or with docetaxel in head and neck cancer. N Engl J Med 2007;357:1705-1715.

23. Jang NY, Wu HG, Park CI, et al. Definitive radiotherapy with or without chemotherapy for $\mathrm{T}_{3}-4 \mathrm{No}$ squamous cell carcinoma of the maxillary sinus and nasal cavity. Jpn J Clin Oncol 2010;40:542-548.

24. Hanna EY, Cardenas AD, DeMonte F, et al. Induction chemotherapy for advanced squamous cell carcinoma of the paranasal sinuses. Arch Otolaryngol Head Neck Surg 2011;137:78-81.

25. Eisenhauer EA, Therasse P, Bogaerts J, et al. New response evaluation criteria in solid tumours: revised RECIST guideline (version 1.1). Eur J Cancer 2009;45:228-247.

26. Edge SB, Byrd DR, Compton CC, Fritz AG, Greene FL, Trotti A. AJCC Cancer Staging Handbook: From the AJCC Cancer Staging Manual. 7th ed. New York: Springer, 2010.

27. Cohen EE, Karrison T, Kocherginsky M, et al. DeCIDE: a phase III randomized trial of docetaxel (D), cisplatin (P), 5-fluorouracil (F) (TPF) induction chemotherapy (IC) in patients with $\mathrm{N}_{2} / \mathrm{N}_{3}$ locally advanced squamous cell carcinoma of the head and neck (SCCHN). J Clin Oncol 2012;30(15 Suppl):5500.

28. Haddad R, O'Neill A, Rabinowits G, et al. Induction chemotherapy followed by concurrent chemoradiotherapy (sequential chemoradiotherapy) versus concurrent chemoradiotherapy alone in locally advanced head and neck cancer (PARADIGM): a randomised phase 3 trial. Lancet Oncol 2013;14:257-264. 
29. Hitt R, Grau JJ, Lopez-Pousa A, et al. A randomized phase III trial comparing induction chemotherapy followed by chemoradiotherapy versus chemoradiotherapy alone as treatment of unresectable head and neck cancer. Ann Oncol 2014;25:216-225.

30. Ghi MG, Paccagnella A, Ferrari D, et al. Concomitant chemoradiation (CRT) or cetuximab/RT (CET/RT) versus induction docetaxel/cisplatin/5-fluorouracil (TPF) followed by CRT or CET/RT in patients with Locally Advanced Squamous Cell Carcinoma of Head and Neck (LASCCHN): a randomized phase III factorial study
(NCTo1086826). J Clin Oncol 2014;32(15 Suppl):6004.

31. Rajapurkar M, Thankappan K, Sampathirao LM, Kuriakose MA, Iyer S. Oncologic and functional outcome of the preserved eye in malignant sinonasal tumors. Head Neck 2013;35:1379-1384.

32. Nishino H, Takanosawa M, Kawada K, et al. Multidisciplinary therapy consisting of minimally invasive resection, irradiation, and intra-arterial infusion of 5-fluorouracil for maxillary sinus carcinomas. Head Neck 2013;35:772-778. 

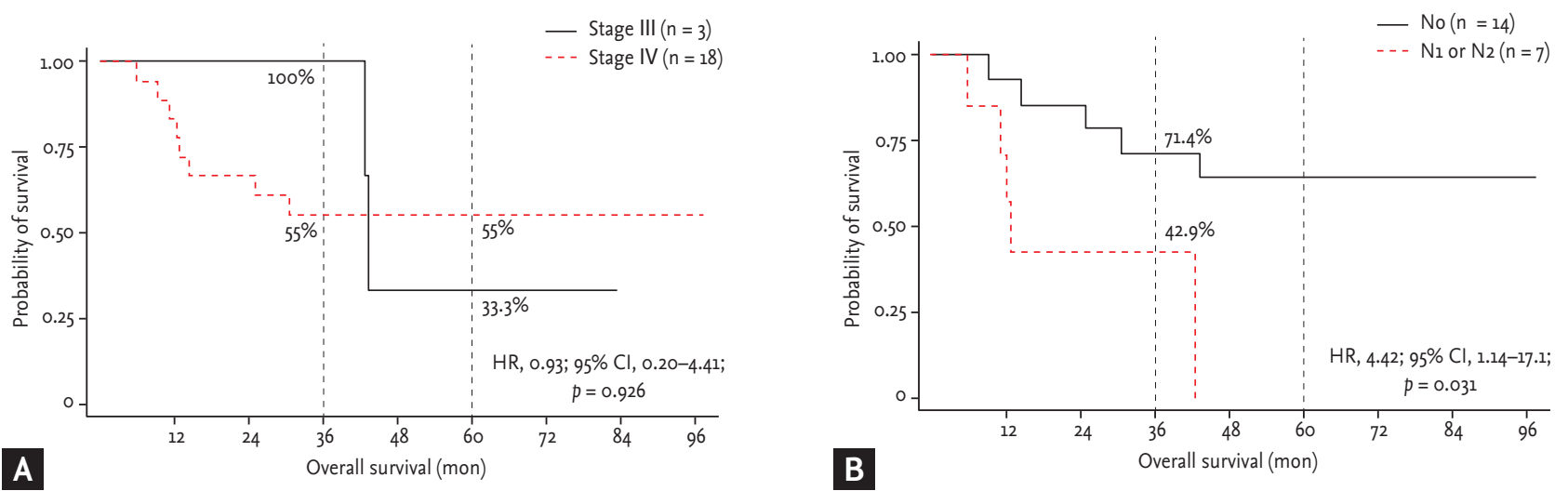

Supplementary Figure 1. Kaplan-Meier curves of patients according to initial stage (A) and N-stage (B). Numbers in the graph indicates probability of survival at 3 years and at 5 years. $\mathrm{HR}$, hazard ratio; CI, confidence interval. 